\title{
Hypohydration produced by high-intensity intermittent running increases biomarkers of renal injury in males
}

\author{
Loris A. Juett ${ }^{1} \cdot$ Katharine L. Midwood $^{1} \cdot$ Mark P. Funnell $^{1} \cdot$ Lewis J. James $^{1} \cdot$ Stephen A. Mears $^{1}$ (D)
}

Received: 24 February 2021 / Accepted: 31 August 2021 / Published online: 15 September 2021

(c) The Author(s) 2021

\begin{abstract}
Purpose Whilst there is evidence to suggest that hypohydration caused by physical work in the heat increases renal injury, whether this is the case during exercise in temperate conditions remains unknown. This study investigated the effect of manipulating hydration status during high-intensity intermittent running on biomarkers of renal injury.

Methods After familiarisation, 14 males (age: $33 \pm 7$ years; $\mathrm{VO}_{2 \text { peak }}: 57.1 \pm 8.6 \mathrm{ml} / \mathrm{kg} / \mathrm{min}$; mean $\pm \mathrm{SD}$ ) completed 2 trials in a randomised cross-over design, each involving 6,15 min blocks of shuttle running (modified Loughborough Intermittent Shuttle Test protocol) in temperate conditions $\left(22.3 \pm 1.0{ }^{\circ} \mathrm{C} ; 47.9 \pm 12.9 \%\right.$ relative humidity). During exercise, subjects consumed either a volume of water equal to $90 \%$ of sweat losses (EU) or $75 \mathrm{~mL}$ water (HYP). Body mass, blood and urine samples were taken pre-exercise (baseline/pre), $30 \mathrm{~min}$ post-exercise (post) and $24 \mathrm{~h}$ post-baseline $(24 \mathrm{~h}$ ).

Results Post-exercise, body mass loss, serum osmolality and urine osmolality were greater in HYP than EU $(P \leq 0.024)$. Osmolality-corrected urinary kidney injury molecule-1 (uKIM-1) concentrations were increased post-exercise $(P \leq 0.048)$, with greater concentrations in HYP than EU (HYP: 2.76 [1.72-4.65] ng/mOsm; EU: 1.94 [1.1-2.54] ng/mOsm; $P=0.003$; median [interquartile range]). Osmolality-corrected urinary neutrophil gelatinase-associated lipocalin (uNGAL) concentrations were increased post-exercise $(P<0.001)$, but there was no trial by time interaction effect $(P=0.073)$.

Conclusion These results suggest that hypohydration produced by high-intensity intermittent running increases renal injury, compared to when euhydration is maintained, and that the site of this increased renal injury is at the proximal tubules.
\end{abstract}

Keywords Renal injury $\cdot$ Water intake $\cdot$ Dehydration $\cdot$ NGAL $\cdot$ KIM-1

\begin{tabular}{|c|c|}
\hline \multicolumn{2}{|c|}{ Abbreviations } \\
\hline (u)KIM-1 & (Urinary) Kidney injury molecule-1 \\
\hline (u)NGAL & $\begin{array}{l}\text { (Urinary) Neutrophil gelatinase-associated } \\
\text { lipocalin }\end{array}$ \\
\hline AKI & Acute kidney injury \\
\hline ANOVA & Analysis of variance \\
\hline ATP & Adenosine triphosphate \\
\hline CK & Creatine kinase \\
\hline CKD & Chronic kidney disease \\
\hline GI & Gastrointestinal \\
\hline LDH & Lactate dehydrogenase \\
\hline LIST & Loughborough Intermittent Shuttle Test \\
\hline Osm & Osmolality \\
\hline
\end{tabular}

Communicated by Fabio Fischetti.

Stephen A. Mears

S.A.Mears@lboro.ac.uk

1 School of Sport, Exercise and Health Sciences, Loughborough University, Leicestershire LE11 3TU, UK

\section{RPE Rating of perceived exertion \\ SD Standard deviation}

\section{Introduction}

The incidence of acute kidney injury (AKI; diagnosed using changes in serum creatinine) following prolonged endurance events (i.e. marathons and ultramarathons) is variable (Lipman et al. 2014; Hoffman and Weiss 2016; Mansour et al. 2017; Poussel et al. 2020), but has been reported to be as high as $85 \%$ (Kao et al. 2015), with some severe cases resulting in temporary dialysis (Hodgson et al. 2017).Whilst these studies are likely to have overestimated AKI incidence, due to issues with measuring serum creatinine in close proximity to exercise (Hodgson et al. 2017), rises in serum creatinine following these events have been accompanied by rises in urinary neutrophil gelatinase-associated lipocalin (uNGAL) and urinary kidney injury molecule-1 (uKIM-1) (McCullough et al. 
2011; Lippi et al. 2012; Mansour et al. 2017; Poussel et al. 2020), which are both novel biomarkers that indicate renal tubular injury (Kashani et al. 2017). The long-term consequences of exercise-associated increases in biomarkers of renal injury are not understood (Hodgson et al. 2017), but there is evidence for a link between AKI and chronic kidney disease (CKD) in a clinical setting (Coca et al. 2012).

The rise in biomarkers of renal injury reported following prolonged endurance events is likely due to several factors, including increases in sympathetic activity and body temperature, as well as muscle damage and hypohydration (Poortmans 1984; Junglee et al. 2013; Hoffman and Weiss 2016; Chapman et al. 2020). Of these factors, hypohydration may be of particular interest in the pathophysiology of exercise-associated renal injury, as whilst it is commonly seen during prolonged endurance events (Cheuvront and Haymes 2001), it can be mitigated with fluid consumption. Doing so may reduce the degree of renal injury, as hypohydration has the potential to contribute to renal injury via multiple mechanisms (Bragadottir et al. 2009), including a reduction in renal blood flow (Smith et al. 1952). It was recently shown that hypohydration produced by simulated physical work in the heat appeared to increase renal injury (compared to when euhydration was maintained with water ingestion) (Chapman et al. 2020). However, though there is observational evidence to suggest that hypohydration may contribute to AKI after a marathon (Mansour et al. 2019), causation cannot be inferred from such data, and thus whether hydration status influences renal injury during exercise in temperate conditions remains unknown and warrants investigation.

Hypohydration also commonly occurs during team sports (Nuccio et al. 2017), where athletes may be at a high risk of renal injury due to the high exercise intensity (Bongers et al. 2017), which can cause a large reduction in renal blood flow (Poortmans 1984) (likely via an increase in sympathetic activity and core body temperature), and may, therefore, lead to ischaemia and subsequent renal injury (Basile et al. 2012). Moreover, the frequent changes of direction and velocity seen in team sports can cause muscle damage (Souglis et al. 2015). Increases in serum creatinine have been measured following soccer matches (Gravina et al. 2011; Colombini et al. 2014), but these increases may be due to the increased release of serum creatinine from damaged skeletal muscle and/or a temporary reduction in renal function, rather than intrinsic renal injury (Hodgson et al. 2017). Therefore, investigation using urinary markers of renal injury is warranted.

The aim of the present study was to investigate the effect of manipulating hydration status during high-intensity intermittent running in temperate conditions on UNGAL and uKIM-1 concentrations. It was hypothesised that hypohydration of $\sim 2 \%$ body mass loss induced by high-intensity intermittent running would result in greater concentrations of UNGAL and uKIM-1, compared to when exercise was completed in a euhydrated state.

\section{Methods}

\section{Subjects}

Fourteen active (team sport/racquet sport players and/or runners) males (age $33 \pm 7$ years; height: $1.79 \pm 0.06 \mathrm{~m}$; body mass: $75.3 \pm 7.6 \mathrm{~kg}$; BMI: $23.6 \pm 2.6 \mathrm{~kg} / \mathrm{m}^{2}$; $\mathrm{VO}_{2}$ peak: $57.1 \pm 8.6 \mathrm{~mL} / \mathrm{kg} / \mathrm{min}$ ) participated in this study. All subjects were healthy, non-smokers, with no previous kidney issues or illnesses. Exclusion criteria included regular use of antiinflammatory medications or any current medical complications that may have impacted kidney function and/or the ability to complete the protocol. Whilst not all participants in the present study were team sport athletes, all participants were familiarised with the exercise protocol, so that it was not a novel stimulus for experimental trials. Ethical approval was granted by the Loughborough University Ethical Approvals (Human Participants) Sub-Committee.

\section{Study design}

Subjects completed a preliminary trial, a familiarisation trial and two experimental trials in a randomised, cross-over design. Trials involved completing a modified version of the Loughborough Intermittent Shuttle Test (LIST) (Nicholas et al. 2000) with (EU trial) and without (HYP trial) water ingestion, followed by a $\sim 20.5 \mathrm{~h}$ recovery period. Familiarisation and experimental trials were separated by $\geq 7$ days.

\section{Preliminary trial}

During this visit, subjects provided verbal and written informed consent, completed a health screen questionnaire and then had height and nude body mass (AFW$120 \mathrm{~K}$, Adam Equipment Co., UK) measured. To determine $\mathrm{VO}_{2}$ peak, subjects then performed an incremental running test on a motorised treadmill (Mercury h/p/cosmos, Nussdorf, Germany), involving sub-maximal and maximal phases. For the sub-maximal phase, the treadmill was initially set at a $1 \%$ incline and $8 \mathrm{~km} / \mathrm{h}$, increasing by $1 \mathrm{~km} / \mathrm{h}$ every 4 min until heart rate exceeded 160 beats/min (Polar M400, Polar, Kempele, Finland). This final speed was used for the maximal phase, where the gradient started at $1 \%$ and increased by $1 \% / \mathrm{min}$, until volitional exhaustion. In the final minute of each sub-maximal stage and for the final minute of the maximal phase, expired gases were collected into a Douglas bag and analysed for oxygen $\left(\mathrm{O}_{2}\right)$ and carbon dioxide $\left(\mathrm{CO}_{2}\right)$ concentrations (Servomex $1400 \mathrm{Gas}$ Analyzer, Servomex, Crowborough, UK), volume (Harvard Dry Gas 
194 Meter, Harvard Apparatus Ltd, Edenbridge, UK) and temperature (RS Pro Digital Thermometer, RS components, Corby, UK). $\mathrm{VO}_{2}$ and $\mathrm{VCO}_{2}$ values were corrected using ambient air collected simultaneously (Betts and Thompson 2012).

\section{Familiarisation trial}

Upon arrival, subjects provided a urine sample and nude body mass was measured, before they completed a standard warm-up on an indoor $20 \mathrm{~m}$ track (two repeats of: $3 \mathrm{~min}$ jogging, followed by three $20 \mathrm{~m}$ sprints, both at a self-selected pace, separated and followed by 2 min rest). Subjects then began the main exercise protocol, which was an adapted version of the LIST (the performance sprints included in a regular LIST were replaced by a cruise at $\sim 95 \% \mathrm{VO}_{2 \text { peak }}$ to reduce trial variability in exercise intensity). This comprised of six 15 min blocks of exercise ( 90 min total) separated by 2 min rest, with a 10 min half-time rest between blocks three and four. Each block was paced using audio cues (Nicholas et al. 2000) and consisted of $\sim 11$ repeated cycles of the following sequence: $3 \times$ walk $(1.5 \mathrm{~m} / \mathrm{s}), 1 \times$ cruise $(\sim 95 \%$ $\mathrm{VO}_{2}$ peak $), 3 \times$ jog $\left(\sim 55 \% \mathrm{VO}_{2}\right.$ peak $)$ and $3 \times$ cruise. Upon completing the adapted LIST, subjects rested in a seated position for $30 \mathrm{~min}$ before a blood sample was taken. Subjects then provided a urine sample and nude body mass was measured. Throughout the familiarisation trial, subjects consumed water ad libitum, which was weighed and added to body mass change (pre-exercise mass minus post-exercise mass) to allow prescription of fluid intake in EU ( $90 \%$ of familiarisation trial sweat losses).

\section{Pre-trial standardisation}

The day prior to their first experimental trial, subjects were instructed to consume at least $40 \mathrm{~mL} / \mathrm{kg}$ body mass of fluid (marked water bottles were supplied to aid compliance) and to complete a $24 \mathrm{~h}$ food/fluid intake diary. Subjects were asked to replicate this in the $24 \mathrm{~h}$ before their second experimental trial. They were also asked to refrain from alcohol intake and strenuous exercise the day before trials. Subjects were sent reminders regarding pre-trial standardisation two days before experimental trials and all subjects confirmed they had adhered to pre-trial requirements upon arrival for experimental trials.

\section{Experimental trials}

Subjects reported to the laboratory between 6 and $10 \mathrm{am}$, after an overnight fast ( $\geq 10 \mathrm{~h}$ without food or fluid). To control diurnal effects, trial start time was standardised within subjects. Upon arrival, subjects sat for $30 \mathrm{~min}$ before a blood sample was taken, during which they completed subjective feelings questionnaires $(0-10$ numbered scale; $0=$ no symptom $; 10=$ maximum symptom) for headache, nausea, dizziness, thirst, gastrointestinal (GI) comfort, GI bloating, stomach fullness and urge to vomit. Thermal sensation was measured on a scale of - 10 (extremely cold) to +10 (extremely hot). After the blood sample, subjects provided a urine sample and their nude body mass was measured, before they consumed $3 \mathrm{~mL}$ water/kg body mass over $15 \mathrm{~min}$. In EU, an additional water bolus was also provided at this time ( $15 \%$ of sweat losses in the familiarisation trial).

Subjects completed the standardised warm-up, then began the modified LIST. In EU, subjects consumed water equivalent to $10 \%$ of their sweat losses from the familiarisation in the rest between the warm-up and the LIST and after each block of the LIST, with an extra 5\% at half-time (15\%). In HYP, to help reduce the unpleasant effects of mouth dryness, subjects consumed $25 \mathrm{~mL}$ water in the rest periods after the first, third and fifth blocks. Heart rate was continuously monitored and then averaged for each block. Rating of perceived exertion (RPE; 6-20), ambient temperature and relative humidity (Kestrel 4400, Nielsen-Kellerman Co, Boothwyn, USA) were measured immediately after each block and subjective feelings questionnaires were completed after block 3 .

Upon completion of the LIST, subjects rested in a seated position for $30 \mathrm{~min}$ and repeated the subjective feelings questionnaires before a blood sample was taken, a urine sample was collected, and nude body mass was measured. Subjects then left the laboratory with food weighing scales and were asked to record their ad libitum food and fluid intake for the remainder of the day. This was subsequently analysed using online software (Nutritics 2019, Dublin, Ireland). The following morning, subjects returned to the laboratory in a fasted state ( $\geq 10 \mathrm{~h}$ fast), $24 \mathrm{~h}$ post-baseline subjective feelings questionnaires were completed, blood/urine samples were collected, and nude body mass was measured.

\section{Sample analysis}

Blood samples were collected by venepuncture of an antecubital vein. From each sample, $1 \mathrm{~mL}$ was dispensed into a tube containing $\mathrm{K}_{2}$ EDTA (1.75 mg/L, Teklab, Durham, UK) and used to determine haemoglobin concentration (cyanmethaemoglobin method) and haematocrit (microcentrifugation; Hawksley Microhematocrit Centrifuge, Hawksley, Worthing, UK), which were used to calculate changes in plasma volume relative to baseline (Dill and Costill 1974). The remaining blood was dispensed into a $5 \mathrm{~mL}$ tube containing $\mathrm{K}_{2}$ EDTA (1.6 mg/L, Sarstedt Ltd, Leicester, UK), which was stored on ice, and a $4.5 \mathrm{~mL}$ tube containing a clotting catalyst (Sarstedt Ltd, Leicester, UK). These were allowed to stand for a minimum of $20 \mathrm{~min}$ before being separated by centrifugation $\left(2200 \mathrm{~g}, 15 \mathrm{~min}, 4^{\circ} \mathrm{C}\right)$. The resulting 
plasma and serum were stored at $-80{ }^{\circ} \mathrm{C}$. All urine samples were measured for volume and osmolality (Osmocheck; Vitech Scientific, Horsham, UK), before being stored at $-80^{\circ} \mathrm{C}$.

Serum samples were thawed and analysed for osmolality, using freezing-point depression (Osmomat Auto, Cryoscopic Osmometer, Gonotec, Berlin, Germany). The concentrations of creatinine, albumin, creatine kinase (CK), lactate dehydrogenase (LDH) and myoglobin in serum samples were determined using a bench-top analyser (ABX Pentra C400; Horiba medical, Northampton, UK). ELISAs were performed for plasma/urinary NGAL (Human NGAL ELISA Kit, BioPorto, Hellerup, Denmark) and urinary KIM-1 (KIM-1 Human ELISA Kit, Enzo Life Sciences, Lausen Switzerland), according to manufacturer's instructions. Intra-assay coefficients of variation for plasma NGAL, uNGAL and uKIM-1 were 8.7, 8.8 and $6.8 \%$, respectively.

\section{Data and statistical analysis}

To control for potential effects caused by shifts in body water, relevant plasma/serum markers were corrected for changes in plasma volume. Furthermore, to control for urine concentration, urinary biomarkers were corrected for urine osmolality. In both cases, uncorrected and corrected data were analysed statistically and presented. Due to an error in data collection, data on subjective feelings questionnaires are presented as $n=12$. Ad-libitum food and fluid intake data are presented as $n=11$, as three subjects did not complete their diet diaries in sufficient detail to be accurately analysed.

Data analyses were performed using SPSS (version 23, SPSS, Chicago, USA). Data were checked for normality of distribution using a Shapiro-Wilk test. Data containing one factor (ad libitum food and fluid intake) were analysed using a paired $t$-test or Wilcoxon signed-rank test, as appropriate. A two-way repeated measures ANOVA was performed to analyse data containing two factors (hydration status $\times$ time; trial order $\times$ time). The Greenhouse-Geisser estimate was used to correct the degrees of freedom where the assumption of sphericity was violated. Significant ANOVA effects were followed up by post hoc paired $t$-tests or Wilcoxon signed-rank tests, as appropriate. Family-wise error rate was controlled using the Holm-Bonferroni adjustment. Datasets were determined to be significantly different when $P \leq 0.05$. Normally distributed data are presented as (mean \pm SD), and non-normally distributed data are presented as [median, interquartile range]. At the time of designing the present study, to our knowledge, there were no published data to inform the effect size of manipulating hydration status on uNGAL or uKIM-1 concentrations. Therefore, a power calculation performed in a prior study, that investigated the effect of muscle damage on uNGAL concentrations, indicated that 12 subjects would be required to detect the smallest relevant change, with a statistical power of 0.8 and an alpha of 0.05 (Junglee et al. 2013). This power calculation is also in agreement with more recently published data (Chapman et al. 2020).

\section{Results}

\section{Trial conditions}

Pre-exercise, body mass (HYP: $74.74 \pm 7.79 \mathrm{~kg}$, EU $74.83 \pm 7.54 \mathrm{~kg} ; P=0.661$ ), serum osmolality (HYP: $292 \pm 2 \mathrm{mOsm} / \mathrm{kgH}_{2} \mathrm{O}$, EU: $292 \pm 3 \mathrm{mOsm} / \mathrm{kgH}_{2} \mathrm{O}$; $P=0.911$ ), haemoglobin (HYP: $15.8 \pm 0.8 \mathrm{~g} / \mathrm{dL}$, EU: $15.9 \pm 0.9 \mathrm{~g} / \mathrm{dL} ; P=0.559$ ), haematocrit (HYP: $43.1 \pm 1.8 \%$, EU $43.3 \pm 1.9 \% ; P=0.739$ ) and thirst sensation (HYP: 4 [3-6], EU 5 [3-6]; $P=0.926$ ) were not different between trials, suggesting a similar hydration status at the start of trials. Ambient temperature (HYP: $22.2 \pm 1.0^{\circ} \mathrm{C}$, EU: $22.3 \pm 0.8{ }^{\circ} \mathrm{C} ; P=0.867$ ) and relative humidity (HYP: $46.1 \pm 14.1 \%$, EU: $49.8 \pm 12.2 \% ; P=0.144$ ) were not different between the trials.

\section{Hydration status measurements}

There was a trial by time interaction effect for changes in body mass (Fig. 1A; $P<0.001$ ), serum osmolality (Fig. 1B; $P<0.001$ ), plasma volume (Fig. $1 \mathrm{C} ; P=0.013$ ) and urine osmolality (Fig. 1D; $P=0.001$ ). Post-exercise, serum osmolality $(P<0.001)$ and urine osmolality $(P=0.024)$ were greater in HYP than EU, but plasma volume was greater in EU than HYP $(P=0.002)$. Sweat loss from preto post-exercise was not different between the trials (HYP: $1.82 \pm 0.25 \mathrm{~kg}$, EU: $1.82 \pm 0.31 \mathrm{~kg} ; P=0.932$ ). However, post-exercise, body mass loss was greater in HYP compared to EU (HYP: $1.6 \pm 0.23 \mathrm{~kg}$, EU: $0.17 \pm 0.22 \mathrm{~kg} ; P<0.001$ ), as $90 \%$ of sweat losses were replaced with water ingestion in EU $(1639 \pm 262 \mathrm{~mL}$; excluding $3 \mathrm{~mL} / \mathrm{kg}$ body mass bolus pre-exercise). In both trials, body mass (Fig. 1A) decreased from baseline to post-exercise $(P \leq 0.020)$ and remained decreased at $24 \mathrm{~h}$ post-baseline $(P \leq 0.033)$. Serum osmolality (Fig. 1B) increased from baseline to post-exercise in HYP $(P<0.001)$ and decreased in EU $(P<0.001)$ but returned to baseline levels at $24 \mathrm{~h}$ in both trials $(P \geq 0.063)$. Plasma volume (Fig. 1C) did not change from baseline to post-exercise in HYP $(P=0.229)$ but increased in $\mathrm{EU}(P<0.001)$. At $24 \mathrm{~h}$, plasma volume was elevated from baseline in both conditions (HYP: $P=0.002$, EU: $P=0.003$ ). Urine osmolality (Fig. 1D) decreased from baseline to post-exercise in EU $(P=0.003)$ but did not change in HYP $(P=0.131)$. At $24 \mathrm{~h}$, urine osmolality was elevated from baseline in both trials $(P \leq 0.004)$. 

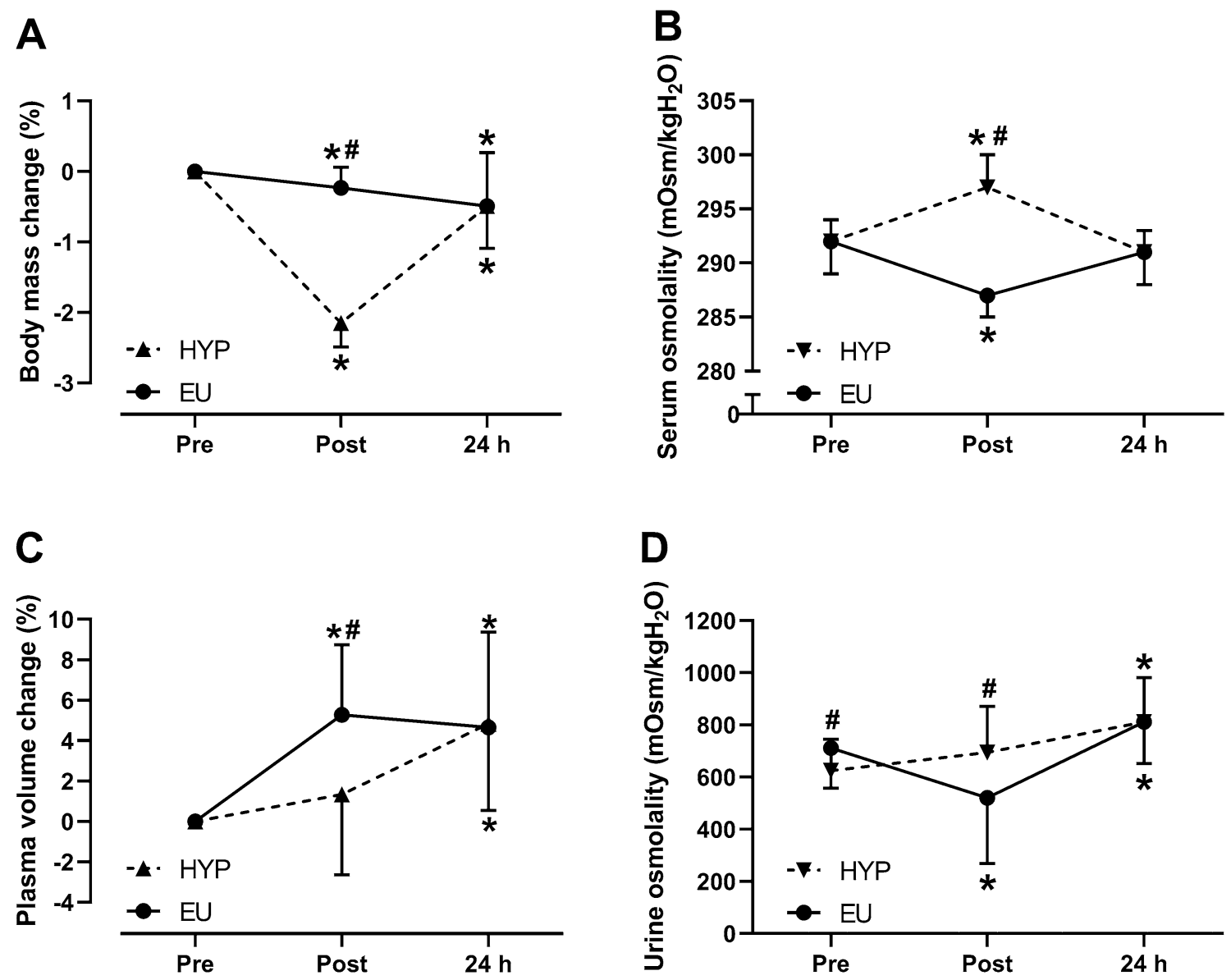

Fig. 1 A change in body mass, $\mathbf{B}$ serum osmolality, $\mathbf{C}$ plasma volume change and $\mathbf{D}$ urine osmolality from pre-exercise (pre/baseline) to 30 min post-exercise (post) to $24 \mathrm{~h}$ post-baseline $(24 \mathrm{~h})$ in HYP $(n=14)$ and EU $(n=14)$. Statistical analyses from two-way repeated

\section{Biomarkers of renal injury}

There were trial by time interaction effects $(P \leq 0.014)$ for uKIM-1 (Fig. 2A) and uNGAL (Fig. 2C) concentrations, with both increased from baseline to post-exercise in HYP (uKIM-1: 2.3-fold increase, uNGAL: 3.4-fold increase; $P=0.002)$ but not in EU $(P \geq 0.300)$. Post-exercise, uKIM-1 and uNGAL were both greater in HYP compared to EU (uKIM-1: $P=0.012$; uNGAL: $P=0.048$ ). At $24 \mathrm{~h}$, uKIM-1 concentrations were elevated from baseline in HYP $(P=0.002)$ but not EU $(P=0.230)$, whereas uNGAL concentrations at $24 \mathrm{~h}$ were not different from baseline in either trial $(P \geq 0.140)$. When correcting uKIM-1 for urine osmolality (Fig. 2B), there was a trial by time interaction effect $(P=0.011)$, with post-exercise osmolality-corrected uKIM-1 $42 \%$ greater in HYP compared to EU $(P=0.003)$. In both trials, osmolality-corrected uKIM-1 increased from baseline to post-exercise (HYP: 2.6-fold increase, EU: 1.6-fold increase; $P \leq 0.048)$, remaining elevated at $24 \mathrm{~h}$ in $\operatorname{HYP}(P=0.030)$ but returning to baseline values at $24 \mathrm{~h}$ in $\mathrm{EU}(P=0.382)$.

measures ANOVA, followed by Holm-Bonferroni-corrected paired $t$-tests. *Significantly different from pre, ${ }^{\#}$ Significant difference between HYP and EU. Data are presented as mean \pm SD

Correcting uNGAL for urine osmolality (Fig. 2D) removed the trial by time interaction effect $(P=0.073)$ but created a time effect $(P=0.015)$, with osmolality-corrected uNGAL increasing from baseline to post-exercise $(P<0.001)$ and returning to baseline values at $24 \mathrm{~h}(P=0.452)$. There was no trial by time interaction effect $(P=0.171)$ for plasma NGAL concentrations (Fig. 2E), but there was a time effect $(P<0.001)$, which increased from baseline to post-exercise $(P<0.001)$, returning to baseline values at $24 \mathrm{~h}(P=0.649)$. Correcting plasma NGAL for plasma volume changes (Fig. 2F) suggested that plasma NGAL remained elevated at $24 \mathrm{~h}(P=0.026)$ but did not alter the significance of any other results.

There were trial by time interaction effects $(P \leq 0.008)$ for serum creatinine (Fig. 3A) and uric acid concentrations (Fig. 3C), which both increased from baseline to postexercise in both trials $(P<0.001)$ and were greater in HYP than EU post-exercise (creatinine: $P=0.015$; uric acid $P=0.042$ ). At $24 \mathrm{~h}$, creatinine concentrations returned to baseline in both trials $(P \geq 0.761)$, whereas uric acid 
A

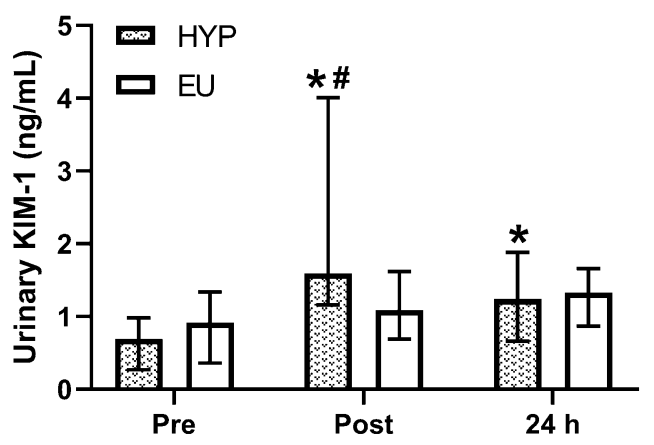

C

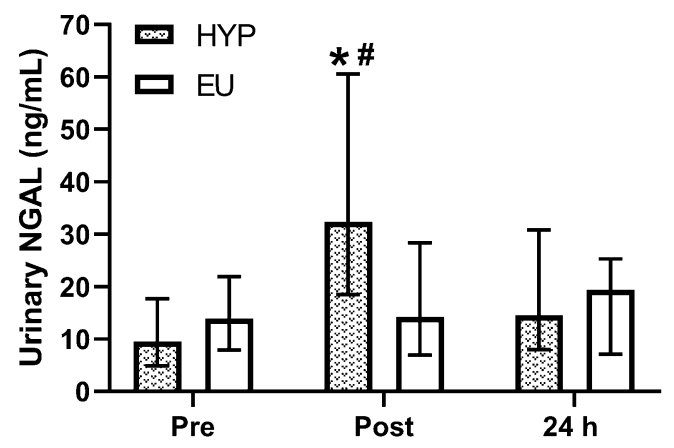

E

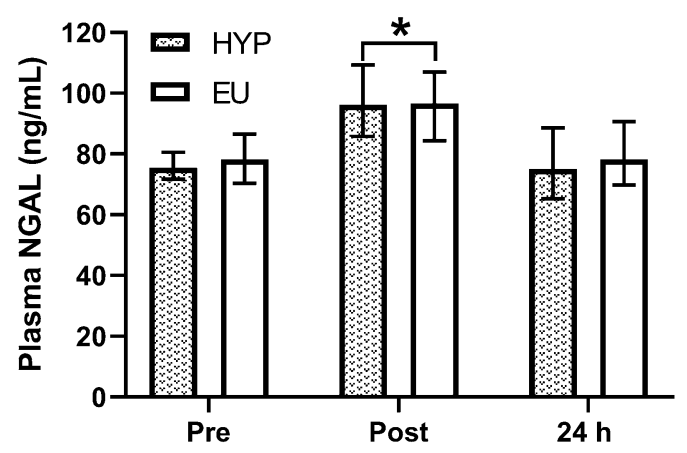

Fig. 2 Urinary KIM-1 (A), osmolality-corrected urinary KIM-1 (B), urinary NGAL $(\mathbf{C})$, osmolality-corrected urinary NGAL (D), raw plasma NGAL (E) concentrations and plasma NGAL concentrations corrected for plasma volume changes $(\mathbf{F})$ before exercise (pre/ baseline), $30 \mathrm{~min}$ post-exercise (post) and $24 \mathrm{~h}$ post-baseline (24 h)

concentrations remained elevated from baseline in both trials $(P \leq 0.030)$. Correcting creatinine (Fig. 3B) and uric acid (Fig. 3D) for plasma volume changes removed the trial by time interaction effect $(P \geq 0.234)$, but the time effect $(P<0.001)$ remained, with plasma volume corrected serum creatinine and uric acid increasing from baseline to post-exercise $(P<0.001)$ and remaining elevated at $24 \mathrm{~h}$ $(P \leq 0.005)$. When investigating effects of trial order, there
B

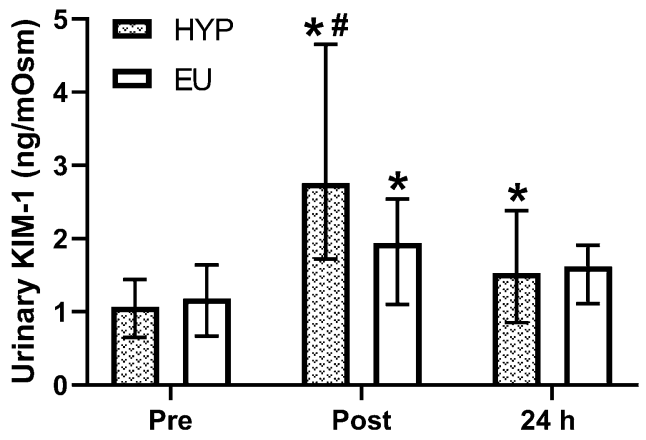

D
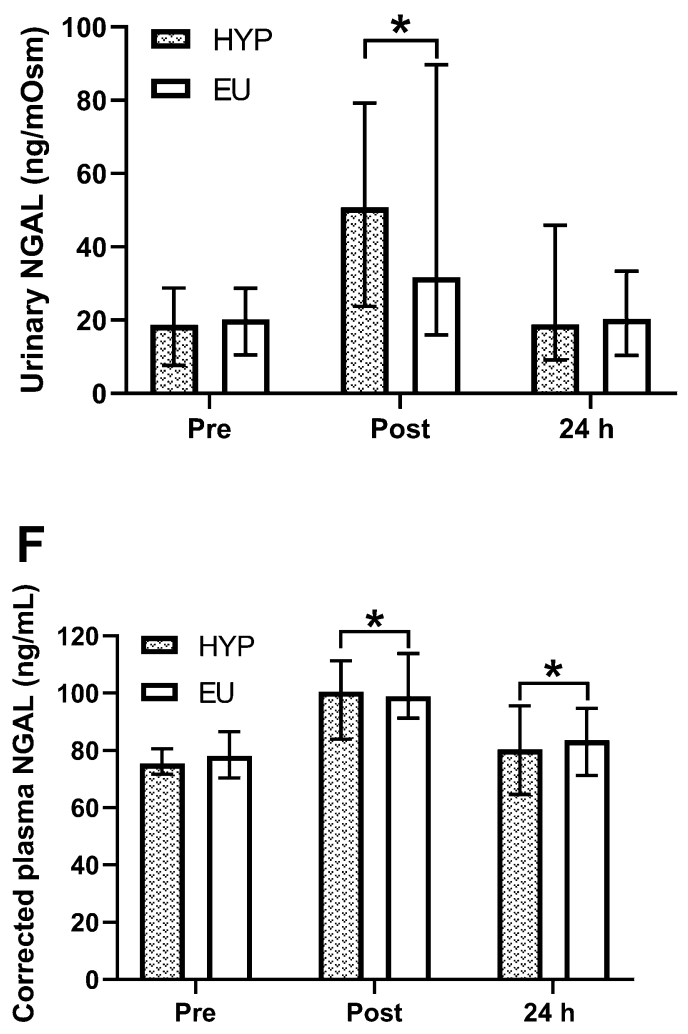

HYP $(n=14)$ and EU $(n=14)$. Statistical analyses from two-way repeated measures ANOVA, followed by Holm-Bonferroni-corrected paired $t$-tests or Wilcoxon signed-rank tests, as appropriate. *Significant difference from pre; "significant difference between HYP and EU. Data are presented as median with interquartile range

were no trial effects $(P \geq 0.108)$ or trial by time interaction effects $(P \geq 0.084)$ for any of the biomarkers of renal injury measured, except plasma volume corrected uric acid (trial by time interaction effect: $P=0.047$ ). However, with regards to plasma volume corrected uric acid, post-hoc tests revealed no significant differences between trials 1 and 2 at any time point $(P \geq 0.123)$, suggesting no effects of trial order on renal injury. 
A

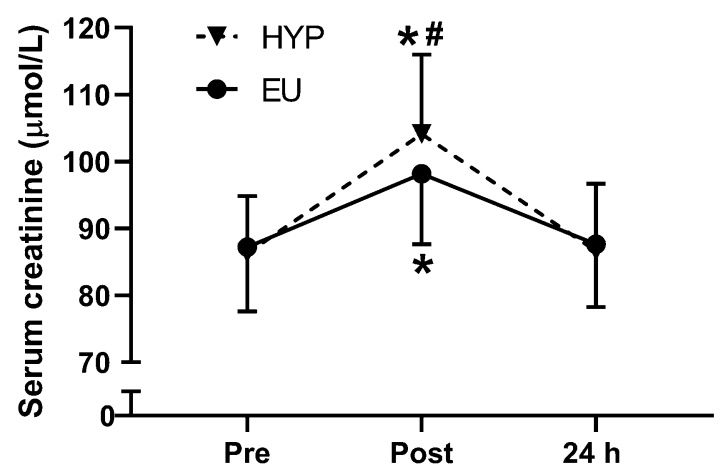

C

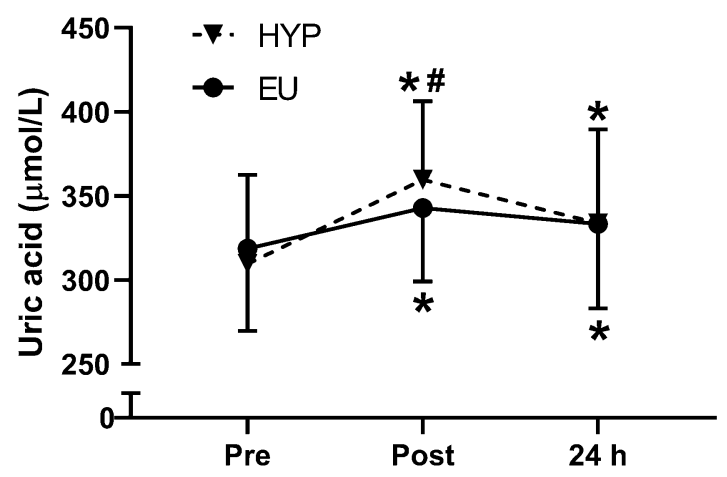

Fig. 3 Raw serum creatinine (A) and serum creatinine corrected for plasma volume changes (B) before exercise (pre/baseline), $30 \mathrm{~min}$ post-exercise (post) and $24 \mathrm{~h}$ post-baseline in HYP $(n=14)$ and EU $(n=14)$. Statistical analyses from two-way repeated measures

\section{Physiological response to exercise and perceptual measures}

There was a trial effect for RPE (HYP: 15, 14-17, EU: 14, 13-15; $P=0.002)$ and heart rate (HYP: $149 \pm 12$ beats/ min, EU: $143 \pm 13$ beats $/ \mathrm{min} ; P=0.004)$. There was a trial by time interaction effect $(P=0.024)$ for serum albumin, which increased from baseline to post-exercise in HYP [765, 745-825 to 804, 781-903, $P=0.004]$ but not EU [779, $745-803$ to $780,766-799, P=0.116]$, with 24 h concentrations not different to baseline in both trials [24 h HYP: 776, 737-818, 24 h EU: 763, 725-818, $P \geq 0.089)$. Post-exercise, serum albumin was greater in HYP than EU $(P=0.003)$.

There was a trial by time interaction effect $(P<0.001)$ for thirst (Table 1), with thirst greater in HYP than EU after block 3 of the LIST $(P<0.001)$ and post-exercise $(P=0.012)$. There was no trial by time interaction effect $(P \geq 0.123)$ for headache, nausea, dizziness or GI bloating (Table 1) and despite a trial by time interaction effect
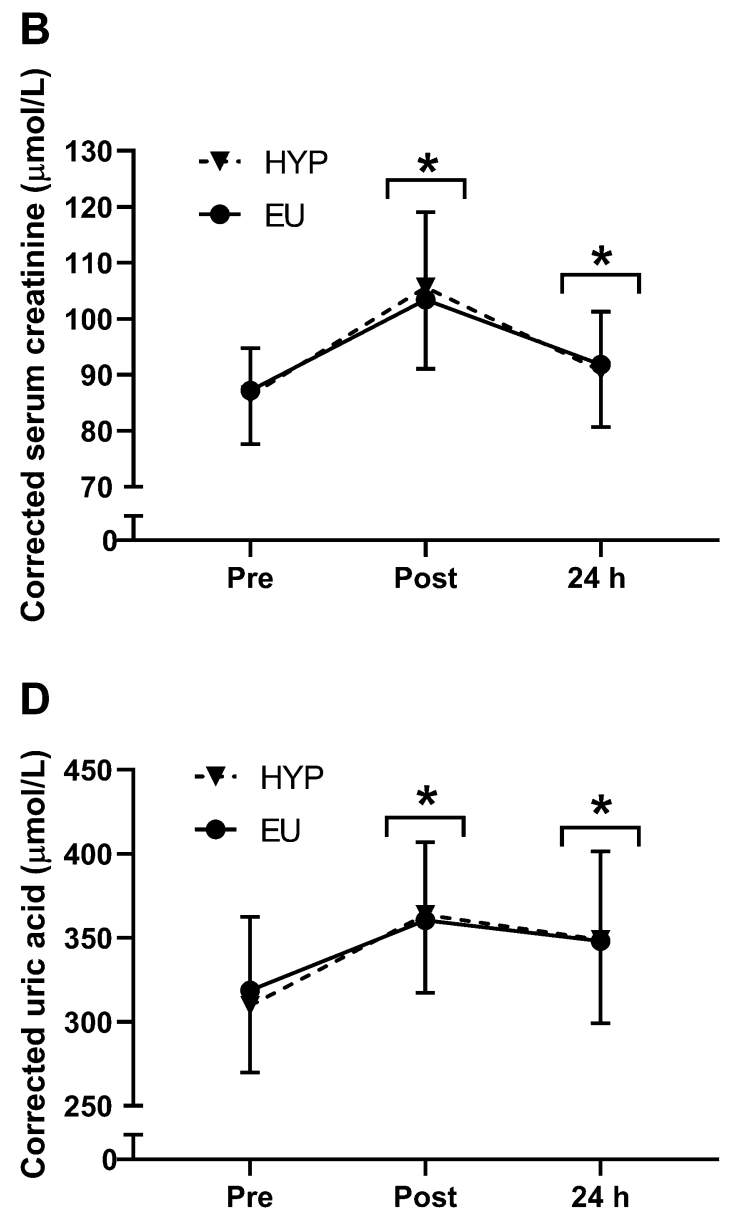

ANOVA, followed by Holm-Bonferroni-corrected paired $t$-tests. *Significant difference from pre; ${ }^{\#}$ significant difference between HYP and EU. Data are presented as mean \pm SD

$(P \leq 0.037)$ for GI comfort, stomach fullness, urge to vomit and thermal sensation (Table 1), post hoc tests revealed no significant differences between trials at any time point $(P \geq 0.056)$.

\section{Muscle damage}

There were no trial effects $(P \geq 0.169)$ or trial by time interaction effects $(P \geq 0.167)$, but there were time effects $(P \leq 0.039)$ for serum myoglobin, $\mathrm{LDH}$ and CK concentrations (Table 2). Serum myoglobin and CK concentrations increased from baseline to post-exercise $(P<0.001)$ and remained elevated from baseline at $24 \mathrm{~h}(P \leq 0.003)$. Correction for plasma volume changes did not alter the significance of any serum myoglobin or CK results. Serum LDH concentration increased from baseline to post-exercise $(P<0.001)$ but returned to baseline values at $24 \mathrm{~h}(P=0.158)$. However, correction for plasma volume changes suggested that $\mathrm{LDH}$ remained elevated from baseline at $24 \mathrm{~h}(P=0.001)$. When 
Table 1 Subjective feelings questionnaires before exercise (pre/baseline), after the third block of the Loughborough Intermittent Shuttle Test (Block $3)$, post-exercise (post) and $24 \mathrm{~h}$ post-baseline in HYP $(n=12)$ and $\mathrm{EU}(n=12)$

\begin{tabular}{|c|c|c|c|c|c|c|c|c|}
\hline & \multicolumn{4}{|l|}{ HYP } & \multicolumn{4}{|l|}{ EU } \\
\hline & Pre & Block 3 & Post & $24 \mathrm{~h}$ & Pre & Block 3 & Post & $24 \mathrm{~h}$ \\
\hline Thirst (0-10) & $4[3-6]$ & $6[5-7]^{\#}$ & $8[6-9]^{\#}$ & $3[2-5]$ & $5[3-6]$ & $2[1-3]$ & $3[2-4]$ & $2[2-5]$ \\
\hline Headache $(0-10)$ & $0[0-0]$ & $0[0-0]$ & $1[0-2]$ & $0[0-0]$ & $0[0-0]$ & $0[0-0]$ & $0[0-0]$ & $0[0-1]$ \\
\hline Nausea $(0-10)$ & $0[0-0]$ & $0[0-1]$ & $1[0-2]$ & $0[0-0]$ & $0[0-0]$ & $0[0-0]$ & $0[0-1]$ & $0[0-0]$ \\
\hline Dizziness (0-10) & $0[0-0]$ & $1[0-2]$ & $1[0-3]$ & $0[0-0]$ & $0[0-0]$ & $0[0-0]$ & $0[0-1]$ & $0[0-0]$ \\
\hline GI bloating (0-10) & $0[0-0]$ & $0[0-0]$ & $0[0-1]$ & $0[0-0]$ & $0[0-0]$ & $1[0-1]$ & $0[0-2]$ & $0[0-0]$ \\
\hline GI comfort $(0-10)$ & $0[0-1]$ & $1[0-1]$ & $1[0-3]$ & $0[0-1]$ & $1[0-1]$ & $0[0-1]$ & $0[0-2]$ & $0[0-0]$ \\
\hline Stomach fullness $(0-10)$ & $0[0-1]$ & $0[0-1]$ & $0[0-0]$ & $0[0-1]$ & $1[0-1]$ & $2[0-2]$ & $1[0-2]$ & $0[0-2]$ \\
\hline Urge to vomit $(0-10)$ & $0[0-0]$ & $0[0-0]$ & $0[0-0]$ & $0[0-0]$ & $0[0-0]$ & $0[0-1]$ & $0[0-0]$ & $0[0-0]$ \\
\hline Thermal sensation ( -10 to 10$)$ & $0[0-0]$ & $4[4-4]$ & $5[4-5]$ & $0[0-0]$ & $0[0-1]$ & $4[4-4]$ & $4[4-5]$ & $0[0-0]$ \\
\hline
\end{tabular}

Statistical analyses from two-way repeated measures ANOVA, followed by Holm-Bonferroni-corrected Wilcoxon signed-rank tests. "Represents a significant difference between trials. Data are presented as median [interquartile range]

Table 2 Myoglobin, lactate dehydrogenase (LDH), LDH corrected for plasma volume changes (C LDH) and creatine kinase (CK) concentrations pre-exercise (pre/baseline), 30 min post-exercise (post) and $24 \mathrm{~h}$ post-baseline $(24 \mathrm{~h})$ in HYP $(n=14)$ and EU $(n=14)$ trials

\begin{tabular}{lllllll}
\hline Biomarker & Pre HYP & Pre EU & Post HYP & Post EU & 24 h HYP & 24 h EU \\
\hline Myoglobin (ng/mL) & $35[25-46]$ & $27[22-45]$ & $139 *[93-465]$ & $141 *[85-239]$ & $50 *[29-77]$ & $42 *[30-53]$ \\
LDH (U/L) & $179[167-184]$ & $169[155-194]$ & $215 *[203-232]$ & $198 *[186-235]$ & $182[167-203]$ & $178[157-197]$ \\
C LDH (U/L) & $179[167-184]$ & $169[155-194]$ & $222 *[209-230]$ & $214 *[205-241]$ & $190 *[171-211]$ & $186 *[166-193]$ \\
CK (U/L) & $292[154-460]$ & $215[105-440]$ & $398 *[237-864]$ & $342 *[198-584]$ & $392 *[255-1040]$ & $428 *[222-699]$ \\
\hline
\end{tabular}

Statistical analyses from two-way repeated measures ANOVA, followed by Holm-Bonferroni-corrected Wilcoxon signed-rank tests. *Represents a significant difference from pre. Data presented as median [interquartile range]

investigating effects of trial order, there were no trial effects $(P \geq 0.168)$ for serum myoglobin, $\mathrm{LDH}$ or $\mathrm{CK}$ and there were no trial by time interaction effects $(P \geq 0.051)$ for $\mathrm{LDH}$ or CK. Despite a trial by time interaction effect $(P=0.033)$ for myoglobin, post hoc tests revealed no significant differences between trials 1 and 2 at any time point $(P \geq 0.165)$, suggesting no effects of trial order on muscle damage.

\section{Food and fluid intake}

Post-exercise energy (HYP: $12,126 \pm 1716$ v EU: $11,871 \pm 2459 \mathrm{~kJ}$ ), protein (HYP: $134 \pm 27 \mathrm{v}$ EU: $133 \pm 40 \mathrm{~g}$ ), fat (HYP: $127 \pm 36 \mathrm{v}$ EU: $114 \pm 43 \mathrm{~g}$ ), carbohydrate (HYP: $303 \pm 69$ v EU: $317 \pm 88 \mathrm{~g}$ ), and sodium (HYP: $2768 \pm 766$ v EU: $2878 \pm 892 \mathrm{mg}$ ) intake were not different between trials $(P \geq 0.345)$. Post-exercise, the intake of water from drinks was greater in HYP $(2997 \pm 843 \mathrm{v}$ $2348 \pm 1166 \mathrm{~g} ; P=0.01$ ), but the intake of water from foods did not differ between trials $(867 \pm 248$ v $928 \pm 210 \mathrm{~g}$; $P=0.444)$. Total water intake for the day was greater in EU $(4165 \pm 902$ v $5180 \pm 1,336 \mathrm{~g} ; P=0.001)$. In HYP, post-exercise ad libitum water intake (including water from foods) was equal to $245 \pm 58 \%$ of post-exercise body mass loss.

\section{Discussion}

The aim of the present study was to investigate the effect of manipulating hydration status during high-intensity intermittent running on biomarkers of renal injury. The main findings were that osmolality-corrected uNGAL and uKIM-1 were both elevated post-exercise, regardless of hydration status, and that post-exercise osmolalitycorrected uKIM-1 was further increased when subjects were hypohydrated. These findings partially confirmed our hypothesis and suggest high-intensity intermittent running causes renal tubular injury and that hypohydration exacerbates this, even in the absence of heat stress. Therefore, this study presents novel data demonstrating that hypohydration exacerbates renal injury after just $90 \mathrm{~min}$ of exercise in temperate conditions (a common place exercise scenario world-wide).

Post-exercise, uKIM- 1 concentrations were greater in the HYP than EUH, despite correction for urine osmolality. This indicates greater production of uKIM-1 in HYP, rather than a simple urine concentration effect, and suggests increased renal tubular injury in HYP. It could be argued that there was a trend for greater post-exercise 
concentrations of osmolality-corrected uNGAL when hypohydrated, but this effect was not significant. Therefore, the differential responses of these biomarkers may provide insight into the location of renal injury that was exacerbated by hypohydration, as KIM-1 expression is increased in response to proximal tubular injury (Ichimura et al. 1998; Han et al. 2002; Kashani et al. 2017), whereas the main contributor to a rise in UNGAL is thought to be an increase in NGAL synthesis in the distal nephron (Paragas et al. 2011; Helanova et al. 2014; Bongers et al. 2017, 2018). However, even if post-exercise osmolality-corrected uNGAL concentrations were significantly greater in HYP, this would not necessarily be indicative of an increase in injury to the distal nephron, as a decrease in the proximal tubular reabsorption of NGAL can also contribute to a rise in uNGAL (Kashani et al. 2017; Schlader et al. 2019). Therefore, these findings suggest that hypohydration produced by high-intensity intermittent running likely exacerbates renal proximal tubular injury. This is in line with previous research by Chapman et al. (2020), which demonstrated that hypohydration produced by $2 \mathrm{~h}$ of simulated physical work in the heat $\left(\sim 39.7{ }^{\circ} \mathrm{C}\right)$ caused an increase (compared to when water was consumed to maintain euhydration) in osmolality-corrected urinary insulin-like growth factor-binding protein 7 , indicating an increase in proximal tubular injury.

In the present study, whilst hypohydration appeared to exacerbate proximal tubular injury, the post-exercise elevations of osmolality-corrected UNGAL and uKIM-1, regardless of hydration status, suggest that the high-intensity intermittent running itself increased renal injury. This was likely due to a reduction in renal blood flow, which was not directly measured in the present study but was evidenced by post-exercise increases in serum uric acid, serum creatinine (although these can be influenced by muscle damage) (Knochel et al. 1974) and plasma NGAL (Schaub and Parikh 2016; Schlader et al. 2019), as well as the previously documented inverse correlation between renal blood flow and heart rate (Poortmans 1984). Given the high-intensity nature of the running in the present study, increases in sympathetic activity (Poortmans 1984; Zouhal et al. 2008) and core body temperature (Radigan and Robinson 1949; Smith et al. 1952; Sato et al. 2019) were likely contributors to a reduction in renal blood flow, which may lead to ischaemia and subsequent renal injury (Basile et al. 2012; Sato et al. 2019). In addition, post-exercise increases in serum myoglobin and uric acid, which were likely caused by muscle damage, may have also contributed to renal tubular injury via a reduction in renal blood flow, as well as other mechanisms (Blomberg et al. 2004; Sánchez-Lozada et al. 2008; Basile et al. 2012; Petejova and Martinek 2014; Roncal-Jimenez et al. 2018). Interestingly, the responses of muscle damage biomarkers in the present study's population were similar to those of elite soccer players following a competitive match (Souglis et al. 2015).

Whilst the high-intensity intermittent running in the present study appeared to increase renal injury (likely via a reduction in renal blood flow), the lack of difference between trials with regards to post-exercise plasma NGAL suggests that the exacerbation of injury to the proximal tubules by hypohydration was not mediated by a further decrease in renal blood flow (Schaub and Parikh 2016; Schlader et al. 2019). The exacerbation of proximal tubular injury by hypohydration may have, therefore, been mediated by the rise in serum osmolality in HYP. This is indicative of intracellular fluid loss, where serum hyperosmolality draws fluid out of the intracellular fluid compartment via osmosis (Cheuvront and Kenefick 2014; James et al. 2019). This triggers the release of arginine vasopressin, which has been shown to increase renal oxygen consumption, possibly exacerbating renal injury by causing renal ischaemia and subsequent renal ATP depletion (Bragadottir et al. 2009; Basile et al. 2012; Cheuvront and Kenefick 2014). Evidence to support this potential mechanism comes from research in mice (RoncalJimenez et al. 2017) and humans, with Mansour et al. (2019) observing that post-exercise copeptin (a stable surrogate for arginine vasopressin) concentrations were greater in marathon runners with AKI than those without AKI.

Typically, an increase in serum osmolality is accompanied by a decrease in plasma volume (James et al. 2019). However, in HYP of the present study, post-exercise plasma volume was not reduced. This may have been due to a high osmotic pressure in the vascular space, as high-intensity exercise has been shown to generate high concentrations of lactate and vasopressin (Mears and Shirreffs 2013). These, combined with hypo-osmotic sweat losses, likely caused the serum hyperosmolality, which appeared to draw fluid from the intracellular compartment to the extracellular compartment (Cheuvront and Kenefick 2014; James et al. 2019). In EU, there was the expected increase in plasma volume as a result of the decreased serum osmolality. There was likely the same osmotic response to exercise, however, the fluid provided, particularly the last bolus, was sufficient to decrease serum osmolality and result in a temporary increase in plasma volume post-exercise.

Though maintaining euhydration during high-intensity intermittent exercise appeared to attenuate proximal tubular injury, it is important to acknowledge that the consumption of large amounts of water can increase the risk of exercise-associated hyponatremia (Rosner and Kirven 2007), evidenced in EU of the current study by a decrease in serum osmolality due the ingestion of plain water. However, no subject's serum osmolality decreased to a concentration that would be deemed as dangerous $(<280 \mathrm{mOsm} /$ $\mathrm{kgH}_{2} \mathrm{O}$; Sahay and Sahay 2014), indicating that the hydration protocol used in EU was safe. This hydration protocol 
was also well tolerated, as evidenced by the lack of difference between trials in perceptual measures related to GI comfort. The hydration protocol also appeared to reduce the physiological strain of the high-intensity intermittent exercise, as measured by the lower heart rates and RPEs in EU, and may therefore have translated into differences in performance if this had been measured (Funnell et al. 2019; James et al. 2019). Whilst the hydration protocol in the present study appeared to attenuate proximal tubular injury, games players will typically drink fluid ad libitum, often consuming less than $50 \%$ of their sweat losses (Garth and Burke 2013; Funnell et al. 2017). Therefore, future research should investigate the effect of this rate of fluid ingestion during exercise on biomarkers of renal injury.

At $24 \mathrm{~h}$ post-baseline, there was evidence to suggest a decrease in body water in both trials, as seen by the lower body mass and increased urine osmolality compared to baseline. It is possible that the lower body mass may be at least partially explained by incomplete glycogen resynthesis, due to impairment of resynthesis by muscle damage (Zehnder et al. 2004) and insufficient carbohydrate intake $(\sim 4 \mathrm{~g} / \mathrm{kg}$ body mass between post-exercise and $24 \mathrm{~h}$ in both trials) (Krustrup et al. 2011). It is not clear whether this is the case, though, as muscle glycogen was not measured in the present study, and studies that have measured muscle glycogen resynthesis following soccer-type exercise have produced contrasting findings (Zehnder et al. 2001; Krustrup et al. 2011).

Even if glycogen resynthesis was incomplete, this would not explain the elevated urine osmolality, which is indicative of a decrease in total body water. This apparent body water deficit at $24 \mathrm{~h}$ is unlikely to be due to an insufficient volume of water consumed in the recovery period, as it was also seen in EU. Moreover, in the $20.5 \mathrm{~h}$ recovery period of HYP, subjects rehydrated with a water volume equal to $245 \%$ of their body mass losses, which is well in excess of the $150 \%$ that has been shown to achieve rehydration in prior studies (Shirreffs et al. 1996; Evans et al. 2017). However, as the majority of the literature has focussed on short-term rehydration $(\leq 6 \mathrm{~h})$ (Evans et al. 2017), the optimal volume of fluid required to achieve rehydration over a longer time period (e.g. $20.5 \mathrm{~h}$ ) remains unknown. Nonetheless, a more likely explanation for the apparent body water deficit at $24 \mathrm{~h}$ is inadequate sodium intake (Sawka et al. 2007). It is recommended that athletes rehydrate with fluid that has a sodium concentration of approximately 40-50 $\mathrm{mmol} / \mathrm{L}$ (Maughan and Leiper 1995; Merson et al. 2008), but in HYP of the present study, when post-exercise sodium intake was expressed relative to total water intake, the concentration was approximately $30 \mathrm{mmol} / \mathrm{L}$, with a similar amount consumed in EU. Therefore, inadequate sodium intake may have resulted in insufficient fluid retention (Maughan and Leiper 1995; Merson et al. 2008; Evans et al. 2017), and thus may explain the apparent body water deficit seen at $24 \mathrm{~h}$ in both trials.

Given that osmolality-corrected uKIM-1 remained elevated at $24 \mathrm{~h}$ in HYP and that there was evidence of a deficit in total body water at $24 \mathrm{~h}$, renal injury was still present and may have been more likely or further increased if another bout of exercise was subsequently performed as is likely for team sports players. However, to our knowledge, the effect of bouts of exercise on consecutive days on uNGAL and UKIM-1 has only been investigated by one study, and whilst these biomarkers did not accumulate after three bouts of exercise performed on consecutive days (Bongers et al. 2017), this exercise was prolonged walking, which likely poses a lower risk of renal injury than more vigorous exercise. Therefore, more research is required to determine the effect of repeated bouts of vigorous exercise on novel biomarkers of renal injury, and the potential influence of hydration status.

In the present study, urine osmolality was used to correct the concentrations of uNGAL and uKIM-1, to account for changes in urine concentration. This is one of a variety of corrections that have been used in this field of research, including urinary creatinine (Junglee et al. 2012; Lippi et al. 2012; Bongers et al. 2017, 2018; Mansour et al. 2019; Chapman et al. 2020; Poussel et al. 2020) and urinary cystatin C (Bongers et al. 2017, 2018). The variety of correction factors used in the literature, along with variations in baseline concentrations (particularly with regards to uKIM-1), mean making comparisons between studies is challenging. Nonetheless, the post-exercise uNGAL concentrations in the present study are lower than those seen after prolonged endurance events (i.e. marathons and ultramarathons), which appear to be the type of exercise that produces the highest post-exercise uNGAL concentrations (Juett et al. 2021). This may be because the average exercise intensity during these events is only slightly lower than the present study, but exercise duration is longer and higher levels of muscle damage appear to be produced (McCullough et al. 2011; Mansour et al. 2019). In the context of exercise-associated renal injury, urine osmolality may be the most appropriate correction factor, as urinary creatinine and cystatin $\mathrm{C}$ may be increased during exercise because of muscle breakdown and decreased proximal tubular reabsorption, respectively (Conti et al. 2006; Junglee et al. 2012; Bongers et al. 2018). Some authors have also corrected urinary kidney injury biomarkers for urine flow rate (Junglee et al. 2013; Chapman et al. 2019, 2020). Whilst it is a limitation of the present study that urine flow rate was not measured, urine osmolality is regarded as an appropriate correction (Bongers et al. 2018; Schlader et al. 2019) and is more likely to become widely utilised, due to difficulties with precisely determining urine flow rate in many settings (Schlader et al. 2019). Another limitation of the present study is that certain mechanistic variables, such 
as core body temperature, renal blood flow and vasopressin/ copeptin, were not measured. However, the significant difference in post-exercise serum osmolality between trials means a difference in vasopressin between trials at this timepoint is extremely likely, as changes in serum osmolality during exercise are strongly correlated with changes in serum arginine vasopressin (Wade 1984).

In conclusion, the results from the present study suggest that high-intensity intermittent exercise may increase renal injury. Maintaining euhydration with water intake during this exercise was a safe and well-tolerated intervention that appeared to attenuate proximal tubular injury and physiological strain. However, the long-term effects of increases in biomarkers of renal injury following exercise are not well understood and should be the focus of future investigations.

Author contributions LAJ, MPF, LJJ and SAM contributed to the design of the study. LAJ, KLM MPF and SAM collected the data, and LAJ, KLM, LJJ and SAM analysed the data. The first draft of the manuscript was written by LAJ. LAJ, LJJ and SAM commented on the versions of the manuscript. All the authors approved the final version of the manuscript.

Funding No external funding was obtained for the current study.

Availability of data and material The datasets generated during the current study are available from the corresponding author on reasonable request.

\section{Declarations}

Conflict of interest LAJ has previously had conference fees covered by Danone Nutricia. LJJ has previously received funding from PepsiCo Inc., Volac International and Entrinsic Beverage Company LLC for hydration-related studies, performed consultancy for PepsiCo Inc. and Lucozade, Ribena Suntory and had conference fees covered by PepsiCo Inc. and Danone Nutricia. In all cases, monies have always been paid to LJJs institution and not directly to LJJ. SAM has previously received funding from the European Hydration Institute and Entrinsic Beverage Company LLC and has had conference fees covered by Danone Nutricia.

Ethical approval This study was performed in line with the principles of the Declaration of Helsinki. Approval was granted by the Loughborough University Ethical Approvals (Human Participants) SubCommittee.

Consent to participate All subjects provided verbal and written informed consent to participate.

Consent for publication Subjects signed informed consent to their data being published.

Open Access This article is licensed under a Creative Commons Attribution 4.0 International License, which permits use, sharing, adaptation, distribution and reproduction in any medium or format, as long as you give appropriate credit to the original author(s) and the source, provide a link to the Creative Commons licence, and indicate if changes were made. The images or other third party material in this article are included in the article's Creative Commons licence, unless indicated otherwise in a credit line to the material. If material is not included in the article's Creative Commons licence and your intended use is not permitted by statutory regulation or exceeds the permitted use, you will need to obtain permission directly from the copyright holder. To view a copy of this licence, visit http://creativecommons.org/licenses/by/4.0/.

\section{References}

Basile DP, Anderson MD, Sutton TA (2012) Pathophysiology of acute kidney injury. Compr Physiol 2:1303-1353. https://doi.org/10. 1002/cphy.c110041

Betts JA, Thompson D (2012) Thinking outside the bag (not necessarily outside the lab). Med Sci Sports Exerc 44:2040. https://doi. org/10.1249/MSS.0b013e318264526f

Blomberg LM, Blomberg MRA, Siegbahn PEM (2004) A theoretical study of myoglobin working as a nitric oxide scavenger. J Biol Inorg Chem 9:923-935. https://doi.org/10.1007/ s00775-004-0585-5

Bongers CCWG, Alsady M, Nijenhuis T et al (2017) Impact of acute versus repetitive moderate intensity endurance exercise on kidney injury markers. Physiol Rep 5:e13544. https://doi.org/10.14814/ phy 2.13544

Bongers CCWG, Alsady M, Nijenhuis T et al (2018) Impact of acute versus prolonged exercise and dehydration on kidney function and injury. Physiol Rep 6:1-11. https://doi.org/10.14814/phy2.13734

Bragadottir G, Redfors B, Nygren A et al (2009) Low-dose vasopressin increases glomerular filtration rate, but impairs renal oxygenation in post-cardiac surgery patients. Acta Anaesthesiol Scand 53:1052-1059. https://doi.org/10.1111/j.1399-6576.2009.02037.x

Chapman CL, Johnson BD, Sackett JR, Parker MD, Schlader Z (2019) Soft drink consumption during and following exercise in the heat elevates biomarkers of acute kidney injury. Am J Physiol Regul Integr Comp Physiol 316:R189

Chapman CL, Johnson BD, Vargas NT et al (2020) Both hyperthermia and dehydration during physical work in the heat contribute to the risk of acute kidney injury. J Appl Physiol 128:715-728. https:// doi.org/10.1152/japplphysiol.00787.2019

Cheuvront SN, Haymes EM (2001) Thermoregulation and marathon running. Sport Med 31:743-762. https://doi.org/10.2165/00007 256-200131100-00004

Cheuvront SN, Kenefick RW (2014) Dehydration: physiology, assessment, and performance effects. Compr Physiol 4:257-285. https:// doi.org/10.1002/cphy.c130017

Coca SG, Singanamala S, Parikh CR (2012) Chronic kidney disease after acute kidney injury: a systematic review and meta-analysis. Kidney Int 81:442-448. https://doi.org/10.1038/jid.2014.371

Colombini A, Machado M, Lombardi G et al (2014) Modifications of biochemical parameters related to protein metabolism and renal function in male soccer players after a match. J Sport Med Phys Fit 54:658-664

Conti M, Moutereau S, Zater M et al (2006) Urinary cystatin C as a specific marker of tubular dysfunction. Clin Chem Lab Med 44:288-291. https://doi.org/10.1515/CCLM.2006.050

Dill DB, Costill DL (1974) Calculation of percentage changes in volumes of blood, plasma, and red cells in dehydration. J Appl Physiol 37:247-248

Evans GH, James LJ, Shirreffs SM, Maughan RJ (2017) Optimizing the restoration and maintenance of fluid balance after exerciseinduced dehydration. J Appl Physiol 122:945-951. https://doi.org/ 10.1152/japplphysiol.00745.2016

Funnell MP, Dykes NR, Owen EJ et al (2017) Ecologically valid carbohydrate intake during soccer-specific exercise does not affect 
running performance in a fed state. Nutrients 9:1-12. https://doi. org/10.3390/nu9010039

Funnell MP, Mears SA, Bergin-Taylor K, James LJ (2019) Blinded and unblinded hypohydration similarly impair cycling time trial performance in the heat in trained cyclists. J Appl Physiol 126:870 879. https://doi.org/10.1152/japplphysiol.01026.2018

Garth AK, Burke LM (2013) What do athletes drink during competitive sporting activities? Sport Med 43:539-564. https://doi.org/ 10.1007/s40279-013-0028-y

Gravina L, Ruiz F, Lekue J et al (2011) Metabolic impact of a soccer match on female players. J Sports Sci 29:1345-1352. https://doi. org/10.1080/02640414.2011.597420

Han WK, Bailly V, Abichandani R et al (2002) Kidney injury molecule-1 (KIM-1): a novel biomarker for human renal proximal tubule injury. Kidney Int 62:237-244. https://doi.org/10.1046/j. 1523-1755.2002.00433.x

Helanova K, Spinar J, Parenica J (2014) Diagnostic and prognostic utility of Neutrophil Gelatinase-Associated Lipocalin (NGAL) in patients with cardiovascular diseases-review. Kidney Blood Press Res 39:623-629. https://doi.org/10.1159/000368474

Hodgson L, Walter E, Venn R et al (2017) Acute kidney injury associated with endurance events-is it a cause for concern? A systematic review. BMJ Open Sport Exerc Med 3:e000093. https://doi. org/10.1136/bmjsem-2015-000093

Hoffman MD, Weiss RH (2016) Does acute kidney injury from an ultramarathon increase the risk for greater subsequent injury. Clin J Sport Med 26:417-422. https://doi.org/10.1016/j.pain.2014.05. 025.Research

Ichimura T, Bonventre JV, Bailly V et al (1998) Kidney injury molecule-1 (KIM-1), a putative epithelial cell adhesion molecule containing a novel immunoglobulin domain, is up-regulated in renal cells after injury. J Biol Chem 273:4135-4142. https://doi. org/10.1074/jbc.273.7.4135

James LJ, Funnell MP, James RM, Mears SA (2019) Does hypohydration really impair endurance performance? Methodological considerations for interpreting hydration research. Sport Med 49:103-114. https://doi.org/10.1007/s40279-019-01188-5

Juett LA, James LJ, Mears SA (2021) Effects of exercise on acute kidney injury biomarkers and the potential influence of fluid intake. Ann Nutr Metab 76:53-59. https://doi.org/10.1159/000515022

Junglee NA, Lemmey AB, Burton M et al (2012) Does proteinuriainducing physical activity increase biomarkers of acute kidney injury? Kidney Blood Press Res 36:278-289. https://doi.org/10. $1159 / 000343417$

Junglee NA, Di Felice U, Dolci A et al (2013) Exercising in a hot environment with muscle damage: effects on acute kidney injury biomarkers and kidney function. Am J Physiol Ren Physiol 305:F813-F820. https://doi.org/10.1152/ajprenal.00091.2013

Kao W-F, Hou S-K, Chiu Y-H et al (2015) Effects of 100-km ultramarathon on acute kidney injury. Clin J Sport Med 25:49-54. https://doi.org/10.1097/JSM.0000000000000116

Kashani K, Cheungpasitporn W, Ronco C (2017) Biomarkers of acute kidney injury: the pathway from discovery to clinical adoption. Clin Chem Lab Med 55:1074-1089. https://doi.org/10.1515/ cclm-2016-0973

Knochel JP, Dotin LN, Hamburger RJ (1974) Heat stress, exercise, and muscle injury: effects on urate metabolism and renal function. Ann Intern Med 81:321-328

Krustrup P, Ortenblad N, Nielsen J et al (2011) Maximal voluntary contraction force, SR function and glycogen resynthesis during the first $72 \mathrm{~h}$ after a high-level competitive soccer game. Eur J Appl Physiol 111:2987-2995. https://doi.org/10.1007/ s00421-011-1919-y

Lipman GS, Krabak BJ, Waite BL et al (2014) A prospective cohort study of acute kidney injury in multi-stage ultramarathon runners: the biochemistry in endurance runner study (BIERS). Res Sport Med 22:185-192. https://doi.org/10.1080/15438627.2014.881824

Lippi G, Sanchis-Gomar F, Salvagno GL et al (2012) Variation of serum and urinary neutrophil gelatinase associated lipocalin (NGAL) after strenuous physical exercise. Clin Chem Lab Med 50:1585-1589. https://doi.org/10.1515/cclm-2011-0954

Mansour SG, Verma G, Pata RW et al (2017) Kidney injury and repair biomarkers in marathon runners. Am J Kidney Dis 70:252-261. https://doi.org/10.1053/j.ajkd.2017.01.045

Mansour SG, Martin TG, Obeid W et al (2019) The role of volume regulation and thermoregulation in AKI during marathon running. Clin J Am Soc Nephrol 14:1297-1305. https://doi.org/10. 2215/cjn.01400219

Maughan RJ, Leiper JB (1995) Sodium intake and post-exercise rehydration in man. Eur J Appl Physiol Occup Physiol 71:311-319. https://doi.org/10.1007/BF00240410

McCullough PA, Chinnaiyan KM, Gallagher MJ et al (2011) Changes in renal markers and acute kidney injury after marathon running. Nephrology 16:194-199. https://doi.org/10.1111/j.1440-1797. 2010.01354.x

Mears SA, Shirreffs SM (2013) The effects of high-intensity intermittent exercise compared with continuous exercise on voluntary water ingestion. Int J Sport Nutr Exerc Metab 23:488-497

Merson SJ, Maughan RJ, Shirreffs SM (2008) Rehydration with drinks differing in sodium concentration and recovery from moderate exercise-induced hypohydration in man. Eur J Appl Physiol 103:585-594. https://doi.org/10.1007/s00421-008-0748-0

Nicholas CW, Nuttall FE, Williams C (2000) The loughborough intermittent shuttle test: a field test that simulates the activity pattern of soccer. J Sports Sci 18:97-104. https://doi.org/10.1080/02640 4100365162

Nuccio RP, Barnes KA, Carter JM, Baker LB (2017) Fluid balance in team sport athletes and the effect of hypohydration on cognitive, technical, and physical performance. Sport Med 47:1-32. https:// doi.org/10.1007/s40279-017-0738-7

Paragas N, Qiu A, Zhang Q et al (2011) The NGAL reporter mouse detects the response of the kidney to injury in real time. Nat Med 17:216-222. https://doi.org/10.1038/nm.2290.The

Petejova N, Martinek A (2014) Acute kidney injury due to rhabdomyolysis and renal replacement therapy: a critical review. Crit Care 18:1-8. https://doi.org/10.1186/cc13897

Poortmans JR (1984) Exercise and renal function. Sport Med Int J Appl Med Sci Sport Exerc 1:125-153. https://doi.org/10.2165/00007 256-198401020-00003

Poussel M, Touzé C, Allado E et al (2020) Ultramarathon and renal function: does exercise-induced acute kidney injury really exist in common conditions? Front Sport Act Living 1:1-7. https://doi. org/10.3389/fspor.2019.00071

Radigan L, Robinson S (1949) Effects of environmental heat stress and exercise on renal blood flow and filtration rate. J Appl Physiol 2:185-191

Roncal-Jimenez CA, Milagres T, Andres-Hernando A et al (2017) Effects of exogenous desmopressin on a model of heat stress nephropathy in mice. Am J Physiol Ren Physiol 312:F418-F426. https://doi.org/10.1152/ajprenal.00495.2016

Roncal-Jimenez CA, Sato Y, Milagres T et al (2018) Experimental heat stress nephropathy and liver injury are improved by allopurinol. Am J Physiol Ren Physiol 315:F726-F733. https://doi.org/10. 1152/ajprenal.00543.2017

Rosner MH, Kirven J (2007) Exercise-associated hyponatremia. Clin J Am Soc Nephrol 2:151-161. https://doi.org/10.2215/CJN.02730 806

Sahay M, Sahay R (2014) Hyponatremia: a practical approach. Indian J Endocrinol Metab 18:760-771. https://doi.org/10.4103/22308210.141320 
Sánchez-Lozada LG, Soto V, Tapia E et al (2008) Role of oxidative stress in the renal abnormalities induced by experimental hyperuricemia. Am J Physiol Ren Physiol 295:F1134-F1141. https:// doi.org/10.1152/ajprenal.00104.2008

Sato Y, Roncal-Jimenez CA, Andres-Hernando A et al (2019) Increase of core temperature affected the progression of kidney injury by repeated heat stress exposure. Am J Physiol Ren Physiol 317:F1111-F1121. https://doi.org/10.1152/ajprenal.00259.2019

Sawka MN, Burke LM, Eichner ER et al (2007) American College of Sports Medicine position stand. Exercise and fluid replacement. Med Sci Sports Exerc 39:377-390. https://doi.org/10.1249/mss. 0b013e31802ca597

Schaub JA, Parikh CR (2016) Biomarkers of acute kidney injury and associations with short- and long-term outcomes. F1000Res. https://doi.org/10.12688/F1000RESEARCH.7998.1

Schlader ZJ, Hostler D, Parker MD et al (2019) The potential for renal injury elicited by physical work in the heat. Nutrients. https://doi. org/10.3390/nu11092087

Shirreffs SM, Taylor AJ, Leiper JB, Maughan RJ (1996) Post-exercise rehydration in man: effects of volume consumed and drink sodium content. Med Sci Sport Exerc 28:1260-1271

Smith JH, Robinson S, Pearcy M (1952) Renal responses to exercise, heat and dehydration. J Appl Physiol 4:659-665

Souglis A, Bogdanis GC, Giannopoulou I et al (2015) Comparison of inflammatory responses and muscle damage indices following a soccer, basketball, volleyball and handball game at an elite competitive level. Res Sport Med 23:59-72. https://doi.org/10.1080/ 15438627.2014.975814

Wade CE (1984) Response, regulation, and actions of vasopressin during exercise: a review. Med Sci Sport Exerc 16:506-511

Zehnder M, Rico-Sanz J, Kühne G, Boutellier U (2001) Resynthesis of muscle glycogen after soccer specific performance examined by $13 \mathrm{C}$-magnetic resonance spectroscopy in elite players. Eur J Appl Physiol 84:443-447. https://doi.org/10.1007/s004210100389

Zehnder M, Muelli M, Buchli R et al (2004) Further glycogen decrease during early recovery after eccentric exercise despite a high carbohydrate intake. Eur J Nutr 43:148-159. https://doi.org/10.1007/ s00394-004-0453-7

Zouhal H, Jacob C, Delamarche P, Gratas-Delamarche A (2008) Catecholamines and the effects of exercise, training and gender. Sport Med 38:401-423. https://doi.org/10.2165/00007256-20083 8050-00004

Publisher's Note Springer Nature remains neutral with regard to jurisdictional claims in published maps and institutional affiliations. 